\title{
Optimizing the Popularity of Twitter Messages through User Categories
}

\author{
Rupert Lemahieu, Steven Van Canneyt, Cedric De Boom and Bart Dhoedt \\ Department of Information Technology \\ Ghent University - iMinds \\ Ghent, Belgium \\ Email: $\{$ steven.vancanneyt,cedric.deboom,bart.dhoedt $\} @$ ugent.be
}

\begin{abstract}
In this paper, we investigate how the category of a Twitter user can be used to better predict and optimize the popularity of tweets. The contributions of this paper are threefold. First, we compare the influence of content features on the popularity of tweets for different user categories. Second, we present a regression model to predict the popularity of tweets given the content features as input. To construct this model, we interpolate a generic regression model, which is trained on all data, and a category-specific model, which is only trained on tweets from users of the same category as the user of the given tweet. In this way we can combine the advantage of the robustness of a generic model, with the ability of categoryspecific models to pick up on category-specific influence of content features. The third contribution is the investigation of the feasibility of boosting the popularity of a tweet by setting up an experiment in which we proactively adapt content features in order to optimize the popularity of tweets. Based on this research, we conclude that the introduction of user categories leads to a more precise analysis and better predictions. In the hands-on experiment, we observed a gain in popularity by proactively adapting content features.
\end{abstract}

Keywords-Twitter, popularity prediction, optimization, user categories.

\section{INTRODUCTION}

Twitter is one of the most used and powerful social media. Nowadays, over 500 million people are registered on Twitter, of which 302 million are active users ${ }^{1}$. This makes Twitter a useful medium for companies to inform users about their products and services. Users, however, are often flooded with Twitter messages, with the consequence that many tweets are read by almost nobody. This can lead to a drop in popularity and even sales of the company.

Therefore, it is important to determine the optimal content of Twitter posts so that they are read, retweeted and replied by many users. For instance, the responses to the tweets typically rise when they contain links to the blog of the company [1]. In addition, the subject of the tweet, the used hashtags, the attached media-such as images, vines, URLs,... - will affect the number of responses [2], [3], [4], [5], [6], [7], [8]. We will study the influence of the content features on a tweet's popularity, as these are most easily adaptable in comparison to other kinds of features. The analysis about this influence, and the prediction of popularity

\footnotetext{
${ }^{1}$ https://about.twitter.com/nl/company
}

using these content features, distinguishes itself from other research by categorizing the Twitter users who post the tweets. We express the popularity in terms of the number of retweets or favorites a tweet receives.

The remainder of this paper is structured as follows. We start with a review of related work in Section II. Next, in Section III, details about the data gathering for analysis and prediction is explained. Section IV describes the analysis of the influence of content features on the popularity of tweets for each considered user category. We build a model to predict the popularity of tweets in Section V. Section VI treats a hands-on experiment in which content features are pro-actively optimized in order to boost popularity. Finally, Section VII concludes this paper.

\section{RELATED WORK}

It has been found that the popularity of a tweet is influenced by some of the tweet's properties [2]. We distinguish a number of feature families. The first ones are the social factors, e.g. the number of followers the user who post the tweet has or the fact if the user is verified or not [3]. Another kind of features are those related to the user history, e.g. the number of tweets placed by the user that were favorited, retweeted or replied to [4]. Next are the timing features, such as the local time of the tweet or the temporal activity of the community [5]. Finally, content features were considered, which include the sentiment value of the tweet [6], hashtags [7], URLs [1], pictures [8] and user mentions [4]. As the content features can most easily adapted for popularity optimization, we will focus on these features in the rest of this paper.

\section{DATA GATHERING AND PREPROCESSING}

We make use of the user categories defined by Socialbakers, a social media analytics company. ${ }^{2}$ They consider 8 user categories and select the users with the most followers in each category. The first two columnn of Table I gives an overview of the categories along with its top user, i.e. the user that has the most followers in that category. From each category, we consider the 500 users that have most followers. For each user, we crawl the 1000 most recent tweetsor less, if the user has not posted 1000 tweets yet. This

\footnotetext{
${ }^{2}$ http://www.socialbakers.com/statistics/twitter/
} 
Table I

OVERVIEW OF THE GATHERED DATA PER USER CATEGORY.

\begin{tabular}{llll} 
Category & Top user Top & \#Users & \#Tweets \\
\hline Brands & SamsungMobile & 500 & 479296 \\
Celebrities & katyperry & 500 & 476448 \\
Community & UberFacts & 500 & 447518 \\
Entertainment & SportsCenter & 500 & 489337 \\
Media & YouTube & 500 & 493197 \\
Place & MuseumModernArt & 500 & 488629 \\
Society & BarackObama & 500 & 481167 \\
Sport & realmadrid & 500 & 494194 \\
\hline Total & & 8000 & 3849786
\end{tabular}

Table II

OVERVIEW OF THE AVERAGE RETWEETS AND FAVORITES PER USER CATEGORY.

\begin{tabular}{lrr} 
Category & Average retweets & Average favorites \\
\hline Brands & 68 & 49 \\
Celebrities & 2112 & 2685 \\
Communities & 828 & 777 \\
Entertainment & 340 & 341 \\
Media & 98 & 59 \\
Places & 25 & 12 \\
Society & 217 & 917 \\
Sport & 99 & 69 \\
\hline Total & 473 & 614
\end{tabular}

gathering process was performed on April 2, 2015 which resulted in 3.8 million tweets. The total number of collected tweets per category are presented in the last column of Table I. The average number of favorites and retweets for each category are shown in Table II.

For each tweet, we retrieve five content features, of which 4 can directly be obtained through the Twitter API. These are the number of user mentions, the number of hashtags, the number of URLs and the number of pictures in the tweet. The fifth feature comprises the sentiment of the tweet, which is expressed with a value between 0 and 1; 0 being neutral and 1 being extremely positive or negative. The sentiment of a tweet is calculated as the average of the sentiment values of its words. These word sentiment values are determined based on the LabMT word list, which contains over 10000 words that were labeled through Amazon's Mechanical Turk [9].

\section{AnAlysis of the Influence of Content FEATURES ON THE POPUlarity OF TWEETS}

In this section, we determine the influence of content features on the popularity of tweets, and how this differs between tweets of different user categories. In particular, for each user category, we want to rank the content features based on their predictive importance towards the tweet popularity. This is performed using LASSO with least angle regression [10]. LASSO is a shrinkage and selection method for linear regression. It minimizes the sum of squared errors, with a bound on the sum of the absolute values of the coefficients. By decreasing the bound, features will be removed from the model as their coefficients take zero
Table III

OVERVIEW OF THE IMPORTANCE OF EACH PREDICTOR FOR THE RETWEETS PER USER CATEGORY USING LASSO.

\begin{tabular}{llllll} 
Category & Pictures & Hashtags & Mentions & URLs & Sentiment \\
\hline Brands & 1 & 2 & 5 & 3 & 4 \\
Celebrities & 4 & 2 & 1 & 3 & 5 \\
Communities & 1 & 2 & 5 & 3 & 4 \\
Entertainment & 2 & 5 & 1 & 3 & 4 \\
Media & 3 & 5 & 2 & 1 & 4 \\
Places & 2 & 3 & 5 & 1 & 4 \\
Society & 5 & 3 & 2 & 1 & 4 \\
Sport & 1 & 3 & 4 & 2 & 5 \\
\hline General & 4 & 3 & 2 & 1 & 5
\end{tabular}

Table IV

OVERVIEW OF THE IMPORTANCE OF EACH PREDICTOR FOR THE FAVORITES PER USER CATEGORY USING LASSO.

\begin{tabular}{llllll} 
Category & Pictures & Hashtags & Mentions & URLs & Sentiment \\
\hline Brands & 2 & 3 & 1 & 4 & 5 \\
Celebrities & 4 & 2 & 1 & 3 & 5 \\
Communities & 1 & 3 & 2 & 4 & 5 \\
Entertainment & 4 & 2 & 1 & 3 & 5 \\
Media & 1 & 4 & 2 & 3 & 5 \\
Places & 2 & 3 & 1 & 4 & 5 \\
Society & 5 & 3 & 1 & 2 & 4 \\
Sport & 2 & 4 & 1 & 3 & 5 \\
General & 4 & 3 & 1 & 2 & 5
\end{tabular}

values. The later the feature is removed from the model, the bigger its predictive importance. The sequence of being removed from the model can thus be used to rank the content features based on their predictive value.

The results when retweets or favorites are considered as tweet popularity measure are listed in Table III and Table IV, respectively. Several conclusions can be drawn from these results. First of all, the sentiment feature has one of the least predictive value of the considered features, as this is consistently ranked fourth or fifth. There are differences between the favorites and the retweets, for instance the user mentions are ranked first or second for the favorites, but obtain for most categories a lower ranking for the retweets. This is because users are more likely to favorite a tweet in which they are mentioned, than to retweet it. We see some different rankings among the user categories, which confirms our presumption that introducing user categories would lead to a more precise analysis. For instance, in case of the retweets, the presence of pictures is ranked first for communities, whereas the presence of a URL is ranked first for the media category. This could be due to the fact that most of the considered communities post funny facts, jokes and memes. For those posts, having a picture in the tweet has a high impact on its popularity. The media mainly contains Twitter accounts of newspapers and other news channels. The most popular tweets that they post contain headlines including a link to their website where the whole article is available to read, leading to a high predictive value of the $U R L$ feature. 
Table V

GENERIC MODEL: OVERVIEW OF CONSIDERED REGRESSION METHODS ALONG WITH THE OBTAINED MSLE ON THE VALIDATION SET.

\begin{tabular}{lr} 
Method & MSLE (Retweets) \\
\hline Ridge Regression & 18.048 \\
Principal Component Regression & 9.844 \\
Partial Least Squares Regression & 9.521 \\
Least Squares Regression & 9.479 \\
Generalized Additive Model & 9.479 \\
Multivariate Adaptive Regression Splines & 8.478
\end{tabular}

Table VI

MSLE VALUES OF THE PREDICTIONS ON THE TEST SET USING THE GENERIC TRAINED MODEL.

\begin{tabular}{lrr} 
& \multicolumn{2}{c}{ MSLE } \\
Category & Retweets & Favorites \\
\hline Brands & 15.136 & 14.207 \\
Celebrities & 8.586 & 12.646 \\
Communities & 5.285 & 8.628 \\
Entertainment & 8.578 & 10.661 \\
Media & 12.083 & 12.340 \\
Places & 5.762 & 7.940 \\
Society & 6.175 & 9.768 \\
Sport & 5.975 & 9.444 \\
\hline Average & 8.448 & 10.704
\end{tabular}

\section{Predicting the Popularity of Tweets}

After analyzing the importance of each content feature to predict the popularity of a tweet, we tackle the problem of predicting the popularity of a tweet, given its features. Therefore, we construct regression models consisting of a generic model and a category-specific model. The models are trained using a training set, containing $50 \%$ of the tweets of each user, optimized using the validation set, containing $25 \%$ of the data, and evaluated using the test set, containing the remaining data. To express performance, the Mean Squared Logarithmic Error (MSLE) is used, which is defined as

$$
\text { MSLE }=\sum_{i=1}^{n}\left(\log \left(1+g\left(x_{i}\right)\right)-\log \left(1+\hat{g}\left(x_{i}\right)\right)\right)^{2}
$$

with $n$ the number of tweets in the test set, $x_{i}$ the observed values for the features of tweet $i, g\left(x_{i}\right)$ the actual number of retweets (resp. favorites) of tweet $i$, and $\hat{g}\left(x_{i}\right)$ the predicted number of retweets (resp. favorites). Note that we also consider the number of follower as a feature in this section, as this feature is a very good predictor of a tweet's popularity [3]. Our approach is explained in more detail in the rest of this section.

\section{A. Generic Model}

The generic model is trained on all data in the training set, without taking into account the user categories. We consider this generic model as the baseline. The validation set is used to test several regression methods, as shown for retweets in Table V. Multivariate adaptive regression splines (MARS) [11] performs best as it has the lowest MSLE of all methods. This regression method is considered in the rest of this paper.
Table VII

MSLE VALUES OF THE PREDICTION ON THE TEST SET USING THE CATEGORY-SPECIFIC MODELS.

\begin{tabular}{lrr} 
& \multicolumn{2}{c}{ MSLE } \\
Category & Retweets & Favorites \\
\hline Brands & 6.419 & 5.168 \\
Celebrities & 12.060 & 14.798 \\
Communities & 6.345 & 7.130 \\
Entertainment & 10.624 & 11.359 \\
Media & 4.533 & 4.770 \\
Places & 4.854 & 2.528 \\
Society & 5.462 & 5.115 \\
Sport & 4.597 & 4.536 \\
Average & 6.862 & 6.925
\end{tabular}

Table VIII

IMPROVEMENT OF THE MSLE VALUES OF THE CATEGORY-SPECIFIC MODELS VERSUS THE GENERIC MODEL.

\begin{tabular}{lrrrr} 
& \multicolumn{2}{c}{ Retweets } & \multicolumn{2}{c}{ Favorites } \\
Category & Absolute & Relative & Absolute & Relative \\
\hline Brands & 8.718 & $57.59 \%$ & 9.039 & $63.62 \%$ \\
Celebrities & -3.475 & $-40.47 \%$ & -2.153 & $-17.03 \%$ \\
Communities & -1.060 & $-20.06 \%$ & 1.498 & $17.36 \%$ \\
Entertainment & -2.046 & $-23.86 \%$ & -0.698 & $-6.54 \%$ \\
Media & 7.551 & $62.49 \%$ & 7.571 & $61.35 \%$ \\
Places & 0.908 & $15.76 \%$ & 5.412 & $68.16 \%$ \\
Society & 0.713 & $11.54 \%$ & 4.653 & $47.63 \%$ \\
Sport & 1.378 & $23.06 \%$ & 4.909 & $51.98 \%$ \\
Average & 1.586 & $18.77 \%$ & 3.779 & $35.30 \%$
\end{tabular}

We present the obtained MSLE values for the prediction on the test set in Table VI. Notice that the prediction errors vary highly: the communities category only has a MSLE of 5.285 for the retweets, while the brands category has a MSLE of 15.136 for the retweets. For all but the brands category, the prediction error in number of retweets is smaller than for the number of favorites.

\section{B. Category-specific Model}

For each considered user category, we constructed a MARS model which is only trained on tweets which are posted by a user of the category. For each tweet in the test set, we first decide to which category it belongs, and then use the corresponding model to predict the number of retweets (resp. favorites). The resulting MSLE values are shown in Table VII. The easiest way to interpret these results is to compare them to the results of the generic baseline model. The improvement of the predictions on the test set of the category-specific models versus the generic model is expressed in Table VIII.

Comparing the MSLE values with the generic model, the category-specific models perform on average better than the generic model. We see that especially the favorites benefit from the introduction of user categories. In a few cases, the category-specific models does not outperform the generic model. A possible explanation could be that the training set is not big enough - the generic model uses a training set 8 times as large-which can result in overfitting the data. 
Table IX

MSLE VALUES OF THE PREDICTION ON THE TEST SET USING THE SMOOTHED MODELS, TOGETHER WITH SMOOTHING PARAMETER $\alpha$.

\begin{tabular}{lrrrr} 
& \multicolumn{2}{c}{ Retweets } & \multicolumn{2}{c}{ Favorites } \\
Category & $\alpha$ & MSLE & $\alpha$ & MSLE \\
\hline Brands & 0.001 & 6.417 & 0.001 & 5.158 \\
Celebrities & 0.969 & 8.429 & 0.987 & 12.567 \\
Communities & 0.993 & 5.265 & 0.009 & 6.917 \\
Entertainment & 1.000 & 8.578 & 0.966 & 10.156 \\
Media & 0.000 & 4.533 & 0.000 & 4.770 \\
Places & 0.835 & 4.634 & 0.000 & 2.528 \\
Society & 0.000 & 5.462 & 0.000 & 5.115 \\
Sport & 0.066 & 4.415 & 0.000 & 4.536 \\
\hline Average & & 5.967 & & 6.468
\end{tabular}

Table X

IMPROVEMENT OF THE MSLE VALUES OF THE SMOOTHED/ MODELS VERSUS THE GENERIC MODEL.

\begin{tabular}{lrrrr} 
& \multicolumn{2}{c}{ Retweets } & \multicolumn{2}{c}{ Favorites } \\
Category & Absolute & Relative & Absolute & Relative \\
\hline Brands & 8.719 & $57.61 \%$ & 9.049 & $63.69 \%$ \\
Celebrities & 0.156 & $1.82 \%$ & 0.078 & $0.62 \%$ \\
Communities & 0.020 & $0.38 \%$ & 1.711 & $19.83 \%$ \\
Entertainment & 0.000 & $0.00 \%$ & 0.505 & $4.73 \%$ \\
Media & 7.551 & $62.49 \%$ & 7.571 & $61.35 \%$ \\
Places & 1.128 & $19.57 \%$ & 5.412 & $68.16 \%$ \\
Society & 0.713 & $11.54 \%$ & 4.653 & $47.63 \%$ \\
Sport & 1.560 & $26.10 \%$ & 4.909 & $51.98 \%$ \\
Average & 2.481 & $29.37 \%$ & 4.236 & $39.57 \%$
\end{tabular}

\section{Smoothed Model}

As neither the category-specific models or the generic model perform best in all categories, a logical next step is to create a new model per category that contains best elements of both: the large training set of the generic model and the specificity of the category-specific model. This is realized through smoothing, in which a weighted version of the category-specific model and the generic model is taken into consideration. If we denote $\mathcal{P}$ as a prediction, the final prediction $\mathcal{P}_{\text {final }}$ can be expressed as:

$$
\mathcal{P}_{\text {final }}=\alpha \cdot \mathcal{P}_{\text {generic }}+(1-\alpha) \cdot \mathcal{P}_{\text {category }}
$$

where $\mathcal{P}_{\text {generic }}$ denotes the prediction by the generic model, $\mathcal{P}_{\text {category }}$ denotes the prediction by the category-specific model and $\alpha \in[0 ; 1]$ the smoothing parameter. The smoothing parameter $\alpha$ is optimized per category on the validation set. The resulting mean squared logarithmic error on the test set are shown in Table IX, along with the smoothing parameters used to obtain these values. To easier interpret these values, we compare the performance of the smoothed models with the generic baseline model in Table X.

On average, in comparison with the generic baseline model, the predictions of the smoothed model improve with almost $30 \%$ for the retweets and $40 \%$ for the favorites. We can distinguish a number of categories which benefit from a large improvement. For instance, an improvement of about $60 \%$ is obtained for the brands and media category, both for number of retweets and favorites. The improvement
Table XI

OVERVIEW OF THE CHOSEN ACCOUNT PER CATEGORY.

\begin{tabular}{ll} 
Category & User \\
\hline Brands & Microsoft \\
Celebrities & katyperry \\
Community & UberFacts \\
Entertainment & hootsuite \\
Media & cnnbrk \\
Place & WaltDisneyWorld \\
Society & BarackObama \\
Sport & nfl
\end{tabular}

is limited for categories such as celebrities, communities and entertainment. One of the reasons is that the tweets of these categories receives on average a lot of retweets and favorites, as shown in Table II. For those tweets, it is harder to improve the mean squared logarithmic error. In general, we can conclude based on our experiments that the introduction of user categories lead to better popularity predictions.

\section{Vi. Proactively Optimizing the Popularity of TwEETS}

As stated above, we focus on content features as these can most easily be adapted to improve the popularity of tweets. To confirm this hypothesis, we investigate to what extent it is possible to boost the popularity of a tweet by proactively changing its content features. For each considered user category, two Twitter accounts are set up. The baseline accounts post tweets with plain text, and the extended accounts post the same text, but with improved content features such as hashtags and pictures. The tweets that are posted, are based on existing Twitter messages that other users already posted. In each category, one user is picked from whom tweets is used in this experiment, these are shown in Table XI.

Firstly, a baseline tweet without any optimized content features is composed from the original tweet. This is done by stripping the tweet of all its entities, such as hashtags, pictures and user mentions. The next step is to construct an optimized version of this baseline tweet. This accomplished by actively optimizing content features, i.e. enriching the baseline tweet with media and entities. For instance, hashtags and pictures are added to enlarge the probability of our tweet being noticed. Both the baseline and the optimized tweet are tweeted by the two different accounts. We illustrate this approach with an example. Figure 1 depicts an original tweet by cnnbrk, Figure 2 shows the baseline tweet based on this tweet and Figure 3 displays the extended tweet.

This experiment was performed between February 2 and March 7, 2015. The total response each category received on the baseline and extended account is listed in respectively Table XII and Table XIII. From these tables, it can be seen that the extended accounts generally receive more interaction, although the differences are small. The advantage of our approach is that the outcome is comparable, since all 
Protests in more than 170 cities last night sparked by \#FergusonDecision. cnn.it/1uG1Fq4

\section{$672 \quad 443$

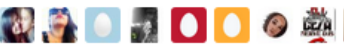 \\ 11:28 AM - 26 Nov 2014}

Figure 1. An original tweet from cnnbrk.

Breaking News

@ThesisNews1

t. +2 Follow

Protests in more than 170 cities last night sparked by FergusonDecision. goo.gl/hhZ6ta

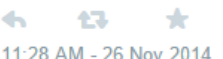

Figure 2. The baseline tweet based on the tweet depicted in Figure 1.

\section{Breaking News}

@ThesisNews2

\section{Protests in more than 170 cities last night} sparked by \#FergusonDecision. goo.gl/AbP7nj \#BreakingNews
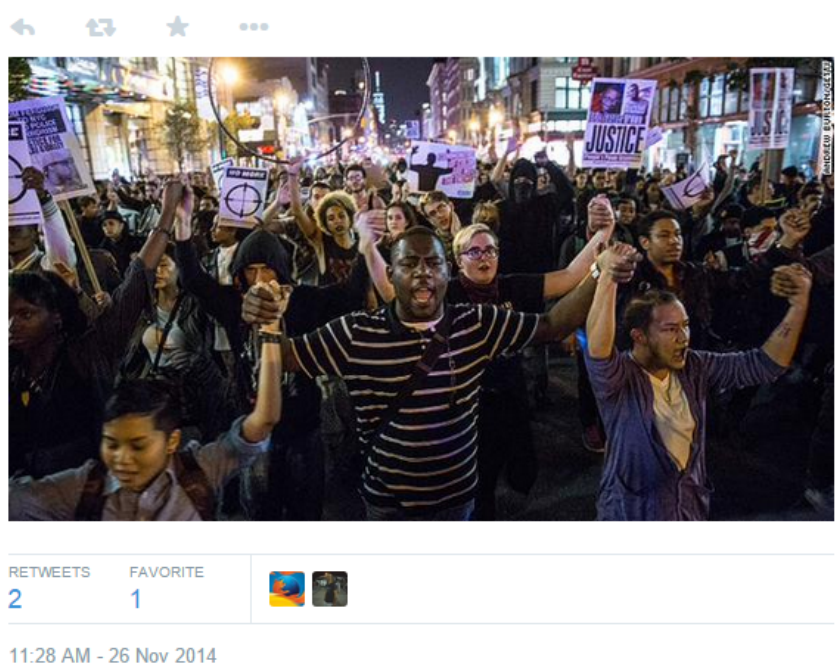

Figure 3. The extended tweet based on the tweet depicted in Figure 1.

accounts start without any followers and post the same, slightly adjusted, tweets. A disadvantage is that because the accounts have no followers, the overall response is small. It might be interesting for the future to conduct this experiment within an actual existing and popular accounts, such as
Table XII

STATISTICS FOR THE BASELINE ACCOUNTS.

\begin{tabular}{llllll} 
Category & Tweets & Followers & Favorites & Retweets & Replies \\
\hline Brands & 96 & 0 & 1 & 0 & 0 \\
Celebrities & 22 & 0 & 0 & 0 & 0 \\
Communities & 2028 & 0 & 8 & 2 & 3 \\
Entertainment & 360 & 1 & 6 & 2 & 2 \\
Media & 533 & 3 & 4 & 4 & 1 \\
Places & 79 & 1 & 2 & 1 & 1 \\
Society & 61 & 0 & 1 & 0 & 0 \\
Sport & 211 & 1 & 19 & 6 & 3
\end{tabular}

Table XIII

STATISTICS FOR THE EXTENDED ACCOUNTS.

\begin{tabular}{llllll} 
Category & Tweets & Followers & Favorites & Retweets & Replies \\
\hline Brands & 96 & 3 & 11 & 10 & 2 \\
Celebrities & 22 & 0 & 0 & 0 & 0 \\
Communities & 2028 & 1 & 11 & 2 & 4 \\
Entertainment & 360 & 9 & 36 & 13 & 4 \\
Media & 533 & 2 & 5 & 7 & 3 \\
Places & 79 & 2 & 6 & 1 & 0 \\
Society & 61 & 0 & 1 & 0 & 0 \\
Sport & 211 & 2 & 7 & 2 & 0
\end{tabular}

cnnbrk. This would probably result in more interaction and gives us a more realistic view of how content features can be adapted to improve the popularity of tweets. Naturally, this would mean that the account needs to agree with the experiment.

\section{CONCLUSION}

We investigated the impact of considering the user category of a tweet to better predict and optimize its popularity. We focused on content features of the tweet, as these are most easily adaptable to improve the popularity of a tweet. For this analysis, we selected users from 8 categories such as brands, celebrities and media. The tweets of these users were first used to compare the impact of content features on the popularity of tweets for the different user categories. Using a method based on LASSO, we concluded that the most important content features differ for different user categories. Second, we constructed a regression method to demonstrate how user categories can be used to improve the prediction of tweet's popularity. Finally, we have conducted an on-hands experiment on Twitter in which we proactively changed content features of a tweet in order to optimize it's popularity. The result of this experiment seems encouraging at this step. Based on this research, we can conclude that making use of user categories is advantageous for analyzing, predicting and optimizing the popularity of tweets. In future work, we will construct a method which first automatically estimates the category of a given user. This will result in a dataset with much more categories and users, and a method that can be used for all Twitter users, even when their categories are unknown. 


\section{ACKNOWLEDGMENT}

Steven Van Canneyt is funded by a $\mathrm{Ph} . \mathrm{D}$. grant of the Agency for Innovation by Science and Technology (IWT). Cedric De Boom is funded by a Ph.D. grant of Ghent University, Special Research Fund (BOF).

\section{REFERENCES}

[1] C. Tan, L. Lee, and B. Pang, "The effect of wording on message propagation: Topic-and author-controlled natural experiments on Twitter," in Proceedings of the 52nd Annual Meeting of the Association for Computational Linguistics, 2014.

[2] B. Suh, L. Hong, P. Pirolli, and E. H. Chi, "Want to be retweeted? Large scale analytics on factors impacting retweet in Twitter network," in Proceedings of the 2nd International Conference on Social Computing, 2010, pp. 177-184.

[3] S. Petrovic, M. Osborne, and V. Lavrenko, "Rt to win! Predicting message propagation in Twitter." in Proceedings of the 5th International Conference on Weblogs and Social Media, 2011, pp. 586-589.

[4] Y. Artzi, P. Pantel, and M. Gamon, "Predicting responses to microblog posts," in Proceedings of the 2012 Conference of the North American Chapter of the Association for Computational Linguistics: Human Language Technologies, 2012, pp. 602-606.
[5] Z. Yang, J. Guo, K. Cai, J. Tang, J. Li, L. Zhang, and Z. Su, "Understanding retweeting behaviors in social networks," in Proceedings of the 19th ACM International Conference on Information and Knowledge Management, 2010, pp. 16331636.

[6] H. Lakkaraju and J. Ajmera, "Attention prediction on social media brand pages," in Proceedings of the 20th ACM International Conference on Information and Knowledge Management.

[7] Z. Ma, A. Sun, and G. Cong, "Will this \#hashtag be popular tomorrow?" in Proceedings of the 35th International ACM SIGIR Conference on Research and Development in Information Retrieval, 2012, pp. 1173-1174.

[8] L. Hong, O. Dan, and B. D. Davison, "Predicting popular messages in Twitter," in Proceedings of the 20th International Conference Companion on World Wide Web, 2011, pp. 57-58.

[9] P. S. Dodds, K. D. Harris, I. M. Kloumann, C. A. Bliss, and C. M. Danforth, "Temporal patterns of happiness and information in a global social network: Hedonometrics and twitter," PloS one, vol. 6, no. 12, pp. 1-26, 2011.

[10] R. Tibshirani, "Regression shrinkage and selection via the lasso," Journal of the Royal Statistical Society. Series B (Methodological), pp. 267-288, 1996.

[11] J. H. Friedman, "Multivariate adaptive regression splines," The Annals of Statistics, pp. 1-67, 1991. 